\title{
Wissenschaftlicher Beirat der ZeFKo
}

Dr. Claudia Baumgart-Ochse, Hessische Stiftung Friedens- und Konfliktforschung (HSFK), Frankfurt a. M.

Prof. Dr. Klaus Boehnke, Bremen International Graduate School of Social Sciences (BIGSSS), Jacobs University Bremen

Prof. Dr. Michael Brzoska, Institut für Friedensforschung und Sicherheitspolitik (IFSH) an der Universität Hamburg Prof. Dr. Susanne Buckley-Zistel, Zentrum für Konfliktforschung (ZfK) der Philipps-Universität Marburg

Prof. Dr. Margit Bussmann, Institut für Politik- und Kommunikationswissenschaft der Ernst Moritz Arndt Universität Greifswald

Prof. Dr. Christopher Daase, Arbeitsbereich Internationale Organisationen der Goethe-Universität Frankfurt am Main, Hessische Stiftung Friedens- und Konfliktforschung (HSFK), Frankfurt a. M.

Prof. Dr. Tobias Debiel, Institut für Entwicklung und Frieden (INEF) der Universität Duisburg-Essen

Dr. Martina Fischer, Berghof Foundation, Berlin

Prof. Dr. Wilhelm Heitmeyer, Institut für interdisziplinäre Konflikt- und Gewaltforschung (IKG) der Universität Bielefeld

Prof. Dr. Hans-Joachim Heintze, Institut für Friedenssicherungsrecht und Humanitäres Völkerrecht der Ruhr-Universität Bochum

Prof. Dr. Martin B. Kalinowski, Zentrum für Naturwissenschaft und Friedensforschung (ZNF) der Universität Hamburg

Dr. Barbara Müller, Institut für Friedensarbeit und gewaltfreie Konfliktaustragung (IFGK), Wahlenau

Prof. Dr. Detlef Nolte, German Institute of Global and Area Studies/Leibniz-Institut für Globale und Regionale Studien (GIGA), Hamburg

Dr. Thania Paffenholz, Centre on Conflict, Development and Peace Building (CCDP) am Graduate Institute of International and Development Studies, Genf

Prof. Dr. Peter Schlotter, Institut für Politische Wissenschaft der Universität Heidelberg

Prof. Dr. Werner Wintersteiner, Zentrum für Friedensforschung und Friedenspädagogik an der Fakultät für Kulturwissenschaften der Alpen-Adria-Universität Klagenfurt

Prof. Dr. Heidrun Zinecker, Institut für Politikwissenschaft der Universität Leipzig

Manuskripte in den drei Rubriken »Aufsätze«, »Literaturbericht« und »Forum« können jederzeit bei der Redaktion der Zeitschrift für Friedens- und Konfliktforschung (zefko@afk-web.de) eingereicht werden. Die in der ZeFKo veröffentlichten Aufsätze und Literaturberichte (max. 10.000 Wörter; inkl. Literaturangaben und Fußnoten) werden einem doppelt anonymisierten Begutachtungsverfahren (double blind peer review) unterzogen. Forums-Beiträge (max. 18.000 Zeichen) werden nicht anonymisiert begutachtet, müssen aber spätestens vier Monate vor dem jeweiligen Erscheinungstermin eines Heftes der Redaktion vorliegen.

Die Rubrik »Aufsätze« bietet die Möglichkeit zur Veröffentlichung der Ergebnisse wissenschaftlicher Forschung in Form methodisch und theoretisch reflektierter Studien zu Fragestellungen der Friedens- und Konfliktforschung. Literaturberichte beschreiben systematisch den Stand der Forschung in einzelnen Themenfeldern und geben Einblicke in wichtige Studien und wissenschaftliche Debatten einzelner Disziplinen oder interdisziplinär bearbeiteter Forschungsbereiche. Forums-Beiträge informieren über aktuelle Entwicklungen in der Friedens- und Konfliktforschung: Im Forum werden vor allem Kommentare und Diskussionsbeiträge zu anderen Veröffentlichungen im Bereich der Friedens- und Konfliktforschung veröffentlicht, aber auch andere Informationen, u.a. Sammelrezensionen zu wichtigen Neuerscheinungen, Hinweise auf Forschungsprogramme oder auch thematisch fokussierte Tagungsberichte.

Aufsatz- oder Literaturbericht-Manuskripte müssen in einer anonymisierten und einer nicht-anonymisierten Version bei der ZeFKo-Redaktion eingehen. Sie dürfen nicht bereits an anderer Stelle veröffentlicht worden sein oder gleichzeitig zur Publikation an anderer Stelle angeboten werden.

Nähere Informationen finden sich auf der Homepage der ZeFKo www.zefko.nomos.de und der AFK www.afk-web.de/ zefko. 


\section{Zeitschrift für}

\section{Friedens- und}

Konfliktforschung

Herausgegeben im Auftrag des Vorstands der Arbeitsgemeinschaft für Friedens- und Konfliktforschung (AFK)

von Thorsten Bonacker (Marburg), Tanja Brühl (Frankfurt a. M.) und Christoph Weller (Augsburg)

\section{Inhalt des 2. Jahrgangs (2013)}

\section{Aufsätze}

\section{Werner Distler/Sarah Riese}

Die andere Seite der Medaille: Intervention und Widerstand in Bosnien-Herzegowina und im Kosovo

Silja Klepp

Europäisierung vor Ort. Eine Ethnographie der Frontex-Mission Nautilus II auf dem

Mittelmeer

Adam Scharpf/Gerald Schneider/Anna Nöh/Aaron Clauset

Die Blutspur des Vetos: Eine Prognose zur Gefahr von extremen Massakern in Syrien

Nicolas Schwank/Christoph Trinn/Thomas Wencker

Der Heidelberger Ansatz der Konfliktdatenerfassung

Ekkart Zimmermann

Der »Arabische Frühling" in vergleichender Revolutionsanalyse

\section{Literaturberichte}

Johanna Ray Vollhardt/J. Christopher Cohrs

Aktuelle sozialpsychologische Beiträge zur Friedens- und Konfliktforschung

Simone Wisotzki

Notfalls mit Gewalt? Globale Gerechtigkeit und die Rechtfertigung militärischer Intervention 


\section{Forum}

\section{Jan Grebe}

Deutsche Rüstungsexporte: Zwischen mangelnder Transparenz, der Notwendigkeit parlamentarischer Kontrolle und einer Neujustierung der Rüstungsexportpolitik

Felix Koltermann

Vom Hier und Dort - Lernen für die Praxis der Feldforschung

Eine Replik auf Susanne Buckley-Zistel

Gert Krell

Zwischen Krieg und Frieden

Eine Auseinandersetzung mit dem Werk von Ekkehart Krippendorff

\section{Editorial}

Tanja Brühl/Thorsten Bonacker/Christoph Weller

Editorial

\section{Abstracts}

$1 / 2013$

$2 / 2013$

290 